\title{
Comparison of three differently shaped ROIs in free breathing breast radiotherapy setup using surface guidance with AlignRT ${ }^{\circledast}$
}

\author{
Marko Laaksomaa ${ }^{1}$, Torsten Moser $^{2}$, Julia Kritz ${ }^{1}$, Kiira Pynnönen ${ }^{1}$, Maija Rossi ${ }^{1,3}$ \\ ${ }^{1}$ Department of Oncology, Tampere University Hospital, Tampere, Finland \\ ${ }^{2}$ VisionRT LTD, London, Great Britain \\ ${ }^{3}$ Department of Medical Physics, Tampere University Hospital, Tampere, Finland
}

\begin{abstract}
Background: Setup accuracy within adjuvant radiotherapy of breast cancer treated in free breathing is well studied, but a comparison of the typical regions of interest (ROI) used in surface guided radiation therapy (SGRT) does not exist. The aim of this study was to estimate the setup accuracy obtained with differently shaped ROls in SGRT.

Materials and methods: A total of 573 orthogonal image pairs were analyzed from free breathing breast patients in two groups: positioning using AlignRT ${ }^{\oplus}$ surface guidance system (Group $A, n=20$ ), and setup using conventional laser and tattoo setup (Group L, $n=20$ ). For SGRT, three different setup ROls were used: a Breast-shaped, O-shaped and T-shaped (B-O and T-ROI). We evaluated the isocenter-, rotation-, pitch and arm position accuracy and residual errors for the chest wall and shoulder joint in $\mathrm{kV}$ orthogonal and tangential setup images with laser- or SGRT-based setup.

Results: Less isocenter variance was found in Group A than in Group L. Rotations and posture errors were larger in group L than in Group A ( $p$ 0.05). Rotation error was smaller with T-shaped ROI than with O- or B-shape $(p=0.01-0.04)$.

Conclusion: Setup with AlignRT ${ }^{\oplus}$ improves reproducibility compared to laser setup. Between the different ROI shapes only small differences were found in the patient posture or the isocenter position in the images. The T-ROI is recommended to set up the chest wall bony structure and an additional B-ROI may be used to fine-tune the soft tissue accuracy.

Key words: breast; surface-guided radiotherapy; region of interest; patient positioning

Rep Pract Oncol Radiother 2021;26(4):545-552
\end{abstract}

\section{Introduction}

Free breathing $(\mathrm{FB})$ radiation therapy for breast cancer, typically with two or three tangential fields, has been used in whole breast (WB) radiation therapy (RT) treatments for decades and is still used in current radiotherapy practice. The low-dose spread is minimized, and treatment implementation is quick and easy with the field in field (FiF) tech- nique [1]. Deep inspiration breath hold (DIBH) technique combined with FiF reduces the dose to the OAR due to a greater extent of the lungs $[2,3]$. $\mathrm{WB}$ radiation therapy in FB is a practical and safe option for elderly patients, right sided breast, and those patients who are unable to achieve $\mathrm{BH}$.

The bony chest wall in tangential images is a reliable estimator for treatment accuracy for tangential treatment fields $[4,5]$. In tangential images, IGRT

Address for correspondence: Marko Laaksomaa, Department of Oncology, Tampere University Hospital, PO BOX 2000 (Teiskontie 35), FI-33521 Tampere, Finland; e-mail: Marko.Laaksomaa@pshp.fi 
thresholds of $5 \mathrm{~mm}$ (cranio-caudal, CC) and $4 \mathrm{~mm}$ (anterior-posterior, AP/lateral, LAT) have been suggested in literature to retain optimal lung dose and to minimize the risk of radiation pneumonitis $[6,7]$. Whilst for tangential treatment it is enough setting up the breast soft tissue roughly inside the treatment field, requirements for VMAT treatment accuracy are typically between 5-8 $\mathrm{mm}$ [8]. The accuracy of the shoulder joint is important in minimizing the risk of shoulder morbidity, which can occur if dose to the shoulder joint is near $15 \mathrm{~Gy}$ [9]. In clinical practice we have noticed that the position of the arm often correlates with the position of the breast. Thereby, a correct arm position is important in WB treatments.

In $\mathrm{WB}$ isocenter setup images (CBCT/2D-2D), typical structures for matching are the sternum for the vertical, the ribs for the lateral, and the equidistance of the ribs and sternum for the longitudinal direction leading to optimal accuracy in the tangential image ribs [6]. With the laser setup, daily isocenter variation at the setup has been reported to be rather large (random error up to $3.1 \mathrm{~mm}$ ) [6] and also arm position correction may be needed based on online images to achieve adequate margin for the humeral head [10]. With SGRT, the isocenter variation is smaller $[11,12]$. Besides, the correct isocenter, patient pitch or rotation may also affect the dose to the heart and lung. These rotations may also be controlled with well-planned SGRT setup.

When using AlignRT ${ }^{\oplus}$ for SGRT, three cameras on the ceiling detect the posture and position of the patient on the treatment couch within sub-millimeter accuracy and compare it with the reference surface generated from the planning CT. The user has to delineate the shape and the size of the region of interest (ROI). For breast treatment, a breast-shaped ROI is recommended. Nevertheless, deformations due to breast swelling [13] or location displacement in the ROI may lead to inaccuracies on the bony chest wall [14]. Therefore, an O-shaped ROI can be used as an alternative in clinical workflows. The O-shaped ROI includes the surrounding area of the breast and excludes the soft tissue. Both ROIs are recommended by VisionRT guidelines [15]. Studies have not previously investigated which ROI leads to the highest accuracy in the relating area, in this case the chest wall.

The aim of this study was to evaluate laser and SGRT setup accuracy and the influence of the shape of SGRT ROIs with DICOM surface. The setup errors in the isocenter and patient posture were measured using orthogonal and tangential $\mathrm{kV}$ images in the radiation therapy of $\mathrm{WB}$ treatments in $\mathrm{FB}$ prior to IGRT.

\section{Materials and methods}

\section{Patient selection and treatment planning}

This study consisted of 40 consecutive patients treated for node-negative breast cancer receiving adjuvant whole breast RT in FB following breast conserving surgery. 20 patients (Group L, mean patient age 59 years) were treated using conventional laser-based setup, 20 patients (Group A, mean age 60) were treated using AlignRT ${ }^{\circledast}$ for setup. Sabella Flex Positioning System (CDR Systems, Canada) was used with a $10^{\circ}$ tilt for patient immobilization (Fig. 1). Patients lifted both arms above the head.

Treatment planning was performed based on computed tomography (CT) imaging using either Philips Brilliance Big Bore (Philips Medical Systems, Eindhoven, The Netherlands) or Toshiba Aquilion LB (Toshiba Medical System, Tokyo, Japan) scanner with $120 \mathrm{kVp}$ and slice thickness of $3 \mathrm{~mm}$. The body was outlined in Eclipse treatment planning system (TPS) (Varian Medical Systems, Palo Alto, CA) using values above -350 Hounsfield Units (HU) for body contour detection. Patients were treated with TrueBeam (Varian Medical Systems, Palo Alto, CA) using a tangential field technique for the whole breast to $45.75 \mathrm{~Gy}$ at 15 or to $42.56 \mathrm{~Gy}$ at 16 fractions due to a change in department conventions for fractionation during the study.

\section{Setup protocol}

Group L: Patients were positioned based on four tattoo marks. Three of the tattoos were at the cross-sectional area from the mammillae (breast), one of them sternally (mid tattoo) and two lateral dorsally from the breast. The fourth tattoo was placed $10-15 \mathrm{~cm}$ caudally from the mid tattoo on the sternum to assist in straightening the patient. The middle tattoo was indexed with the fixation at the CT and was used at setup. At the treatment device, the patients were positioned to the tattoos with couch at zero laterally and rotationally. Then, the couch was shifted automatically to the planned isocenter position using Delta Couch function (Varian Medical Systems, Palo Alto, CA). 


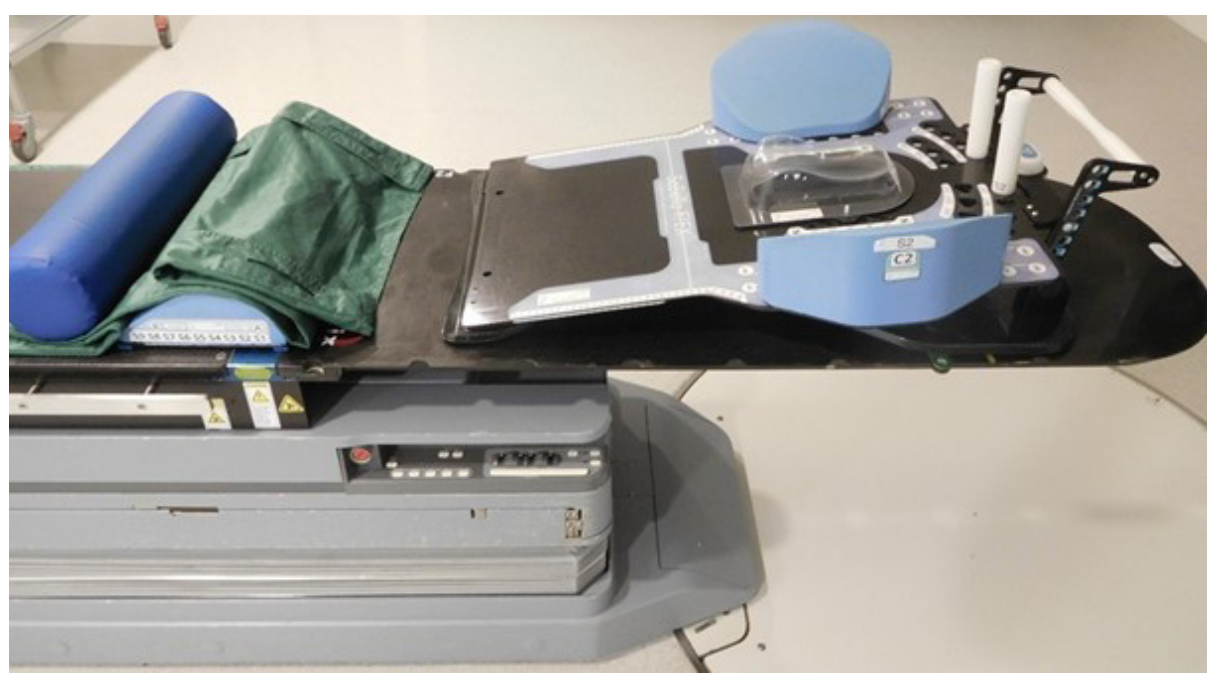

Figure 1. Sabella fixation was used in this study. For the arm position verification with SGRT device is optimal, because there is several individual adjustments and elbow supports are not blocking the cameras, and the entire arm is visible

Group A: Patients were positioned based on the AlignRT $^{\circledast}$ DICOM reference surface. Rotations were corrected based on displayed values. Patient setup tolerance was $1 \mathrm{~mm}$ for translations, $1^{\circ}$ for ROLL/ROT and $2^{\circ}$ for pitch. When the rotations were inside thresholds, the AlignRT ${ }^{\circledast}$ move couch function was used to shift the couch automatically to the SGRT-based isocenter in all three directions. The arm position was verified with a treatment capture.

\section{ROls in AlignRT ${ }^{\oplus}$}

Three different ROIs were used in this study. First, a breast-shaped ROI (B-ROI) was drawn as the whole breast (Fig. 2A) on the PTV side. Second, a ROI similar to the B-ROI was drawn but excluding an $\mathrm{O}$-shaped soft tissue region (O-ROI) (Fig. 2B). Third, an ROI shaped as a T upside-down was drawn (T-ROI) (Fig. 2C) including the diaphragm bilaterally, the sternum, and a small portion of the breast tissue bilaterally.

The ROI patterns were alternated for the setup, using each every third day during 15 (or 16) fractions. On the first fraction, patient setup was performed with T-ROI, on the second day with the $\mathrm{O}-\mathrm{ROI}$ and on the third day with the B-ROI. Thus, each setup ROI was used at least five times with one patient.

\section{IGRT protocol}

Verification $\mathrm{kV}$ images were acquired with TrueBeam system (TrueBeam, Varian Medical Systems, Palo Alto, CA) at $80 \mathrm{kV}$ and $8-10.4 \mathrm{mAs}$ for anterior images, at $95 \mathrm{kV}$ and $8-16 \mathrm{mAs}$ for lateral images and at $70 \mathrm{kV}$ and $2.2 \mathrm{mAs}$ for the tangential images. The isocenter correction was based

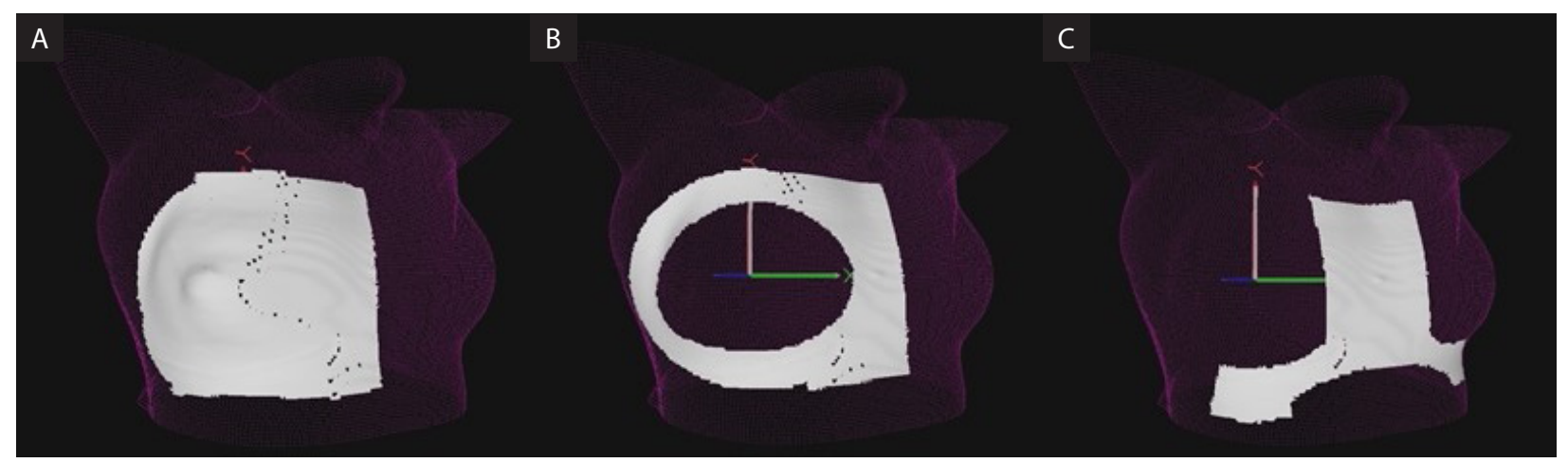

Figure 2. In this study we used three different kinds of ROIs. A. Breast-shaped ROI (B-ROI); B. O-shaped soft tissue region (O-ROI); C. ROI shaped as T upside-down (T-ROI) 


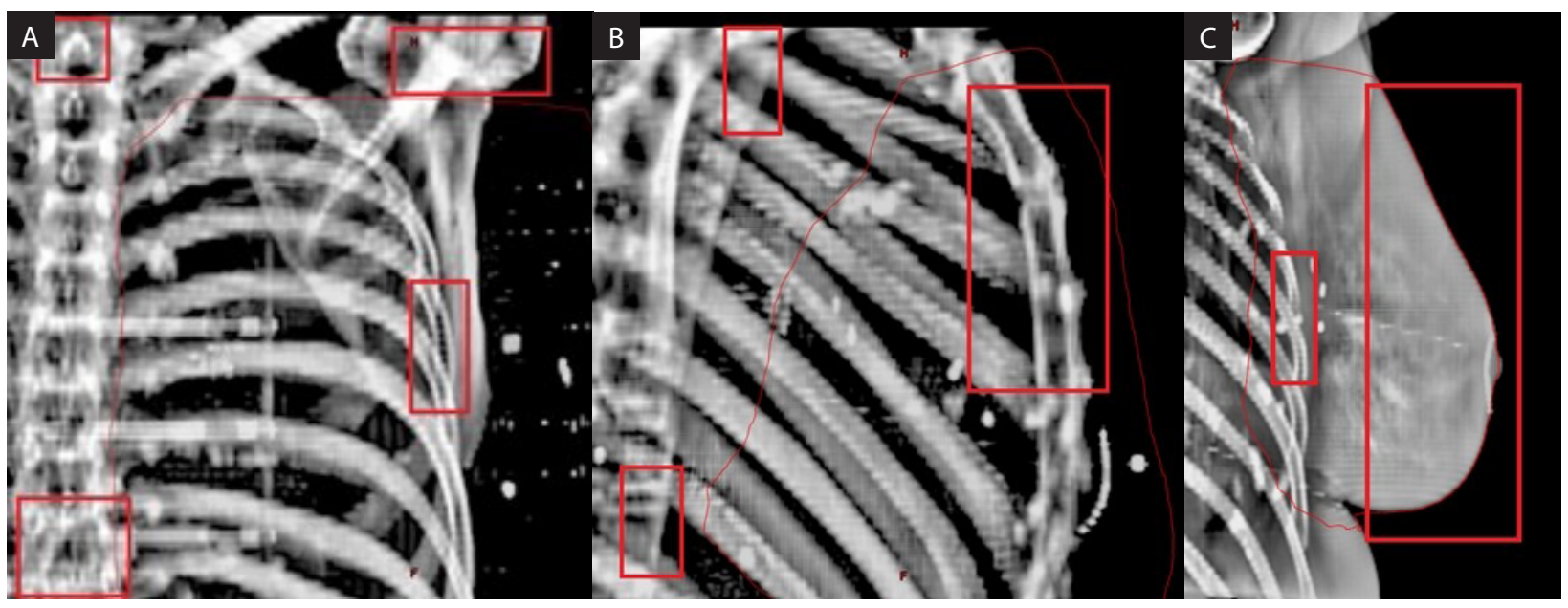

Figure 3. Evaluated landmarks for evaluation of the errors were $\mathrm{TH} 1$ and th8/10, ribs and shoulder joint in the AP-image (A), th1 and th8/10, sternum in the LAT-image $(\mathbf{B})$ and ribs and the soft tissue $(\mathbf{C})$ in the tangential image

on daily orthogonal setup images. Translational couch corrections were performed based on the online isocenter match of pre-defined landmarks at the chest wall, sternum and ribs (Fig. 3). Rotation corrections were only performed when the posture error of the patient vertebra exceeded $1^{\circ}$ in the online match. Arm position was corrected if the shoulder joint near the PTV was shifted more than $7 \mathrm{~mm}$ towards PTV. Daily (Group A) or weekly (Group L) tangential breast images were acquired after couch corrections to verify the location of treatment. After couch shifts AlignRT screenshot was acquired to evaluate the differences with DICOM.

\section{Offline image analysis}

The images $(n=900)$ were matched retrospectively by an experienced radiotherapist to exclude interobserver variability. The images were matched to the th1, th8-10, sternum, the ribs and shoulder joint, individually for each landmark (Fig. 3). To evaluate the displacement between the mid-vertebra and sternum, the mid-th was retrospectively calculated as average of th 1 and th8/10. The isocenter was defined in the offline image matching to the midway compromise of the ribs and the sternum (AP, LAT, SI). In the tangential images, the residual errors of the ribs and breast tissue were evaluated only in the SI direction. The residual errors in the images were added to the isocenter couch corrections based on online match. These all analyses thus demonstrate the setup accuracy prior to daily IGRT with different
ROIs of the SGRT and with laser setup. In addition to residual errors in shifts, positional errors in the rotation (th1-th10), pitch (mid-vertebra-sternum) and arm position (th1-shoulder joint) (Figure 3) were evaluated.

\section{Statistical analysis}

The setup accuracy was compared between Group A and Group L. In addition, the B-ROI, O-ROI and T-ROI were evaluated separately. Two-tailed F-test was applied for systematic errors (test for equality of variances). The Wilcoxon rank sum test was applied for random errors (test for equality of means). A p-value $\leq 0.05$ was considered statistically significant.

\section{Results}

\section{Isocenter}

Table 1 shows the agreement between orthogonal $\mathrm{kV}-\mathrm{kV}$ images and Laser- or SGRT-setup for $\mathrm{AP}, \mathrm{SI}$ and LAT to the isocenter. For translations, especially the AP direction showed a considerable decrease of both systematic and random errors in Group A compared to Group L $(p=0.006)$ and systematic errors were smaller in Group A in the LAT direction ( $p=0.01-0.15$ ). Moreover, the number of outliers was smaller in Group A compared to Group L. If the same acquired couch value was used with laser setup daily, no difference was found between Groups A and L in the sternum in the AP direction $(p=0.15-0.39)$. The mean residual error in the AP direction (i.e. sternum) was $-1.8 \mathrm{~mm}$ 
Table 1. Residual isocenter errors for the AlignRT with DICOM surfaces or with laser setup (mm). For the laser setup two values are given, first for the DeltaCouch analysis, second for couch vertical acquired after three fractions as in the department protocol

\begin{tabular}{|l|c|c|c|}
\multicolumn{2}{|c|}{ AP } & SI & $\mathbf{2 . 5}(\mathbf{L}) \pm 2.1$ \\
\hline B-ROI & $\mathbf{1 . 5}\left(\mathbf{L}^{*}\right) \pm \mathbf{2 . 0}\left(\mathbf{L}^{*}\right)$ & $3.0 \pm 2.2$ & $2.8 \pm 2.3$ \\
\hline O-ROI & $\mathbf{1 . 7}\left(\mathbf{L}^{*}\right) \pm \mathbf{1 . 8}\left(\mathbf{L}^{*}\right)$ & $\mathbf{1 . 8}(\mathbf{B}, \mathbf{L}) \pm \mathbf{2 . 1}$ (L) & $\mathbf{2 . 1}$ (L) \pm 2.2 \\
\hline T-ROI & $\mathbf{1 . 8}\left(\mathbf{L}^{*}\right) \pm \mathbf{1 . 6}\left(\mathbf{L}^{*}\right)$ & $2.1 \pm \mathbf{2 . 0}(\mathbf{L})$ & $4.0 \pm 2.7$ \\
\hline Laser & $4.3 / 1.2 \pm 3.3 / 1.9$ & $3.0 \pm 2.7$ & \\
\hline
\end{tabular}

The bolded values are significantly better $(p<0.05)$ than what is indicated in parenthesis. *The comparison to Group $L$ was significant only with DeltaCouchShift not with acquired couch vertical. $\mathrm{ROI}$ - region of interest; B-ROI - breast-shaped ROI; O-ROI - O-shaped soft tissue region; T-ROI - ROI shaped as T upside-down

Table 2. Residual errors between the bony structures $(\mathrm{mm})$ without couch corrections. TH1-TH8/10 (vertebra rotation and pitch), TH8/10- sternum (chest wall pitch) and TH1-shoulder (arm position)

\begin{tabular}{|c|c|c|c|c|}
\hline \multirow{5}{*}{ 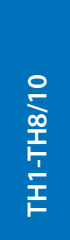 } & & AP & SI & LAT \\
\hline & B-ROI & $0.7(L) \pm 0.8(L)$ & $0.8 \pm 0.8$ & $2.8 \pm 3.3$ \\
\hline & O-ROI & $0.8(L) \pm 0.8(L)$ & $0.6 \pm 1.0$ & $2.5 \pm 2.8$ \\
\hline & T-ROI & $0.6(L) \pm 0.8(L)$ & $0.7 \pm 0.7$ & $2.3 \pm 2.2(\mathrm{~B}, \mathrm{O})$ \\
\hline & Laser & $1.4 \pm 1.3$ & $0.6 \pm 1.0$ & $2.2 \pm 2.7$ \\
\hline \multirow{4}{*}{ 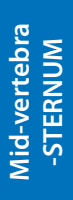 } & $\mathrm{B}-\mathrm{ROI}$ & $1.0(L) \pm 1.1(L)$ & $1.6(\mathrm{~L}) \pm 1.6(\mathrm{~L})$ & \\
\hline & O-ROI & $\mathbf{1 . 0}(\mathrm{L}) \pm 1.2$ & $1.4(\mathrm{~L}) \pm 1.6(\mathrm{~L})$ & \\
\hline & T-ROI & $0.9(\mathrm{~L}) \pm 1.3$ & $1.3(\mathrm{~L}) \pm 1.3(\mathrm{~L})$ & \\
\hline & Laser & $1.7 \pm 1.4$ & $2.3 \pm 2.2$ & \\
\hline \multirow{4}{*}{ 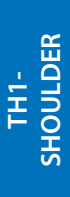 } & B-ROI & & $2.2 \pm 2.3$ & $2.6 \pm 1.7$ \\
\hline & O-ROI & & $2.4 \pm 2.4$ & 1.5 (B) \pm 1.5 \\
\hline & T-ROI & & $1.8 \pm \mathbf{2 . 1}(\mathrm{L})$ & $1.6(B) \pm 1.6$ \\
\hline & Laser & & $2.8 \pm 3.0$ & $2.1 \pm 1.9$ \\
\hline
\end{tabular}

The bolded values are significantly better $(p<0.05)$ than what is indicated in parenthesis; ROI — region of interest; $\mathrm{B}-\mathrm{ROI}$ - breast-shaped ROI; $\mathrm{O}-\mathrm{ROI}$ - O-shaped soft tissue region; $\mathrm{T}-\mathrm{ROI}$ - $\mathrm{ROI}$ shaped as $\mathrm{T}$ upside-down

(O-ROI), $-2.6 \mathrm{~mm}$ (T-ROI), $-2.0 \mathrm{~mm}$ (B-ROI) in Group A and $-2.3 \mathrm{~mm}$ in Group L, patient sternum remaining too dorsally in the images compared to DRR. After couch shifts, in Group A the DICOM vertical (VRT) delta had mean value between +0.8 and $+1.3 \mathrm{~mm}$ in the screenshots. In the SI direction, T- and O-ROIs improved when compared to Group L ( $\mathrm{p}=0.001-0.10)$. Within Group A, the systematic error was smaller with O-ROI than with B-ROI $(\mathrm{p}=0.02)$. In the LAT direction, the T-ROI and B-ROI had lower systematic error than Group L.

\section{Residual errors}

Table 2 shows patient rotation based on difference between th 1 and th8/10. In Group A the lateral random rotation with $\mathrm{T}$-ROI was smaller than with B- and O-ROIs ( $\mathrm{p}=0.005-0.038)$. The systematic and random pitch of the vertebra was larger within
Group L compared to Group A ( $\mathrm{p}=0.0001-0.05$ in systematic, $0.001-0.04$ in random).

Table 2 also shows postural errors between structures that cannot be corrected with $3 \mathrm{D}$ isocenter shift but only with repositioning. In the difference between the mid-vertebra and sternum, Group A was better than Group L in the SI direction in both systematic and random errors $(\mathrm{p}=0.01-0.04)$, and in most cases in the AP direction. The smallest variance was found in T-ROI, especially with respect to minimum and maximum values and outliers. The displacement between TH1 and the shoulder joint showed slightly lower systematic $(p=0.06)$ and random ( $\mathrm{p}=0.05)$ offset for T-ROI in the SI direction in comparison to Group L (Tab. 2). Among the ROIs, the T- and O-ROIs were better than the B-ROI in systematic LAT errors $(\mathrm{p}=0.02-0.03)$. The random errors in Group A were below $2.4 \mathrm{~mm}$ in the SI direction and below $1.7 \mathrm{~mm}$ in the LAT 
Table 3. Residual errors for the AlignRT ${ }^{\circledR}$ with DICOM surfaces or with laser setup [mm] in the LNG direction

\begin{tabular}{|c|c|c|}
\hline & Chest wall & Soft tissue \\
\hline B-ROI & $3.1 \pm 2.4$ & $1.2(\mathrm{~T}, \mathrm{~L}) \pm 1.5(\mathrm{~T}, \mathrm{~L})$ \\
\hline O-ROI & $2.2 \pm 1.9$ (L) & $1.8(\mathrm{~L}) \pm 1.8(\mathrm{~L})$ \\
\hline T-ROI & $2.4 \pm \mathbf{2 . 0}(\mathrm{L})$ & $2.4 \pm 2.2$ \\
\hline Laser & $3.2 \pm 2.7$ & $3.3 \pm 2.6$ \\
\hline
\end{tabular}

$\mathrm{B}-\mathrm{ROI}$ - breast-shaped region of interest; The bolded values are significantly better $(p<0.05)$ than what is indicated in parenthesis

direction, and in Group L they were $3.0 \mathrm{~mm}$ (SI) and $1.9 \mathrm{~mm}$ (LAT).

\section{Tangential images}

In the tangential images (Tab. 3), the O-ROI and T-ROI were better than Group L in the random SI direction in the chest wall ( $\mathrm{p}=0.03$ for T-ROI, 0.01 for O-ROI). For the soft tissue the B-ROI was better than T-ROI or Group L, and the O-ROI was better than Group L (Tab. 3).

\section{Discussion}

\section{Isocenter accuracy with laser or AlignRT ${ }^{\oplus}$ setup}

In this study we evaluated the influence of differently shaped ROIs on setup accuracy of WB treatments in FB. The isocenter reproducibility was higher for the SGRT setup, in all of the ROIs. If, however, the systematic error in the DICOM surface is large, acquiring a new reference surface can assist in reducing isocenter errors in the images and possibly reduce the need for daily isocenter images. In our experience, acquiring the reference surfaces for the setup and thereby replacing the DICOM reference needs a well-established workflow and, therefore, well-trained staff and offline review evaluation. With laser setup, isocenter accuracy was slightly lower than with SGRT setup; however, acquiring the couch vertical reduced the difference in the AP direction.

In Group A, only two sternal marks were used to align the patient to the middle of the breast board on the centered couch. The AlignRT ${ }^{\circledast}$ DICOM reference surface was then used to further correct rotational and translational setup errors. Russell et al. found no difference between the groups regarding the errors on the chest wall in tangential images when AlignRT ${ }^{\oplus}$ setup was done with or without initial tattoo setup [16]. The results of this study are thus likely applicable to using markerless RT and AlignRT ${ }^{\circledast}$.

Hattel et al. [17] reported no improvements in the SI direction or rotation in comparison to laser setup using a similar B-ROI as in this study. In the AP direction even more accurate alignment was found with lasers than with SGRT. Our study agreed in the SI direction, but in the AP direction SGRT with B-ROI was more reproducible than laser setup with the DeltaCouch function (Tab. 1, 2). The matching locations in the AP direction are different between the studies (vertebra [17] vs. sternum in this study). However, if acquired couch vertical value was used in group L, no difference was found between the Group A and Group L, indicating that couch VRT should not be shifted with the delta couch function with laser setup, but instead the same couch value in the VRT direction should be used daily. Besides the B-ROI, we found a higher reproducibility for T-ROI and partly for O-ROI than for laser setup in all directions.

Sáa et al. found that the largest discrepancy between $\mathrm{CBCT}$ data and AlignRT ${ }^{\oplus}$ B-ROI occurred in the SI direction and in pitch [18]. Also in our data with B-ROI, rather large systematic isocenter error was found in the SI direction. Alderliesten et al. found as well that the errors are the largest in the SI direction using B-ROI, and they found no improvements for larger chest wall ROI covering both breasts. This was considered typical for the SGRT, as soft tissue variation can be caused for example by the arm position in this region. Additionally, the breast tissue is deformable and shape changes are possible $[13,19]$, leading to discrepancy between soft tissue and chest wall. This discrepancy was also noticeable in this study in the tangential images in the SI direction and in the orthogonal images with larger needs to shift the isocenter in the SI direction with B-ROI.

In all groups there was on average a $2-\mathrm{mm}$ need to raise the couch in the VRT direction to match the sternum. In Group L this can be explained with different bending of the couch between CT and treatment. For Group A, however, the SGRT detects the surface, and treatment couch bending should be accounted for by the system. There are two possible explanations for this. First, the patient chest wall may relax systematically between patient positioning and image acquisition [20]. Second, the HU selection for body outline detection in the planning system may be suboptimal, leading to sys- 
tematic errors between the body contours detected by SGRT and defined in the DICOM. To avoid the effect of relaxation, the move couch in the VRT direction should be done just before image acquisition, at the time deltas are not at zero. There was 0.8-1.3 mm systematic difference between IGRT and the AlignRT delta values of the screenshots after IGRT. To avoid this, the limit of HU-value for body detection could be adjusted; or small external margin to BODY could be created at the TPS for SGRT. This might aid in better isocenter accuracy in the DICOM surface.

\section{Displacement between the structures}

The T-ROI reflected the vertebral rotation the best. This rotation may have an influence on the heart and lung dose, but it is difficult to evaluate patient rotation with tangential images. The random error in rotation of $2.2 \mathrm{~mm}$ for the T-ROI was slightly more reliable than for other ROIs with 2.8-3.3 $\mathrm{mm}$. Postural errors are more difficult to correct at initial set up based on laser marks only and, therefore, pitch-related errors were more pronounced in Group L than in Group A, even when limiting actions are taken, such as indexing the middle tattoo in order to standardize patient longitudinal position on the fixation device. Errors will influence total lung volume radiated; in some cases a decreased distance between the vertebra and sternum as a consequence of pitch errors was seen. This can lead to a discrepancy in the planned dose to the heart [21].

With an optimal breast board for SGRT WB setup, the entire arm is visible in the treatment capture and fixation itself should not be blocking the cameras in the arm area from any side. In the CT data only limited information on arm position is visible, as the DICOM ends typically up to chin. Therefore, a new reference surface can be acquired for the setup to include the correctly positioned arm. Thus, positioning is easier and possibly even more accurate. When the arm position was verified with treatment capture using AlignRT, this led to 2.1-2.4 $\mathrm{mm}$ random error in all the groups in the SI direction, which is smaller when compared to data found in the literature with laser setup $(3.6 \mathrm{~mm})$ [6], or with SGRT and the arm-ROI (3.5 mm) [22]. In the current study with the Sabella fixation device (Fig. 3), the arm was better aligned with AlignRT ${ }^{\circledast}$ compared to laser setup.

\section{Setup accuracy in the tangential images}

Setup accuracy was evaluated in the chest wall and soft tissue for the SI direction in Group A in tangential images. Accuracy on the chest wall was better in T-ROI and O-ROI than in B-ROI. However, the B-ROI was more accurate for the soft tissue. The setup may thus first be initiated with the T-ROI to achieve optimal alignment of the bones, and then the B-ROI can be used as confirmation on the soft tissue. Previous literature reports this mismatch of the errors between the chest wall and soft tissue. It may be possible to acquire new setup surfaces with both soft tissue and chest wall in their correct location to reduce the systemic mismatch errors $[13,14,19]$. With laser setup, the reproducibility of the tangential image was on the same level when compared to the weakest ROIs for the chest wall or soft tissue in the SI direction, specifically when using the B-ROI for the chest wall and T-ROI for the soft tissue. AlignRT shows better reproducibility with properly selected ROIs and reference surfaces compared to laser set-ups in node-negative patients.

In the literature, $\mathrm{CBCT}$ is recommended for VMAT treatments due to the decreased allowance for soft-tissue deformations compared to tangential treatments [8]. In addition to CBCT, it is possible to use SGRT with B-ROI to detect changes in skin contour with no added radiation.

\section{Conclusion}

Using AlignRT ${ }^{\oplus}$ as a primary setup tool, for all ROIs investigated, smaller variances were found compared to conventional laser based setup. The TROI led to the smallest uncertainties, especially in patient rotation, ensuring that the planned dose is correctly delivered to the PTV, and no excess dose is caused to the heart and lung. The B-ROI can be used additionally to verify the breast position at the setup to ensure accurate PTV coverage, especially in VMAT treatments.

\section{Conflict of interest}

Torsten Moser is working for VisionRT.

\section{Funding}

The authors have received no funding or grants for this work. 


\section{Acknowledgements}

The authors wish to thank Menna Creed for kindly language editing the paper.

\section{References}

1. Hegazy EA. Dosimetric comparison of Field in Field (FiF) and three dimensional planning techniques (3D) used in treatment of breast cancers. Glob J Phys. 2019; 9(1): 813-820.

2. Pandeli C, Smyth LML, David S, et al. Dose reduction to organs at risk with deep-inspiration breath-hold during right breast radiotherapy: a treatment planning study. Radiat Oncol. 2019; 14(1): 223, doi: 10.1186/s13014-0191430-x, indexed in Pubmed: 31822293.

3. Essers M, Poortmans PM, Verschueren K, et al. Should breathing adapted radiotherapy also be applied for right-sided breast irradiation? Acta Oncol. 2016; 55(4): 460-465, doi: 10.3109/0284186X.2015.1102321, indexed in Pubmed: 26503610.

4. Batumalai V, Phan P, Choong C, et al. Comparison of setup accuracy of three different image assessment methods for tangential breast radiotherapy. J Med Radiat Sci. 2016; 63(4): 224-231, doi: 10.1002/jmrs.180, indexed in Pubmed: 27741383.

5. Smith RP, Bloch P, Harris EE, et al. Analysis of interfraction and intrafraction variation during tangential breast irradiation with an electronic portal imaging device. Int J Radiat Oncol Biol Phys. 2005; 62(2): 373-378, doi: 10.1016/j. ijrobp.2004.10.022, indexed in Pubmed: 15890577.

6. Laaksomaa M, Kapanen M, Skyttä T, et al. Estimation of optimal matching position for orthogonal $\mathrm{kV}$ setup images and minimal setup margins in radiotherapy of whole breast and lymph node areas. Rep Pract Oncol Radiother. 2014; 19(6): 369-375, doi: 10.1016/j.rpor.2014.05.001, indexed in Pubmed: 25337409.

7. Aiello D, Borzì G, Marino L, et al. Comparison of deep inspiration breath hold and free breathing technique in left breast cancer irradiation: a dosimetric evaluation in 40 patients. J Radiat Oncol. 2019, doi: 10.1007/s13566019-00381-3.

8. Rossi M, Boman E, Skyttä T, et al. Dosimetric effects of anatomical deformations and positioning errors in VMAT breast radiotherapy. J Appl Clin Med Phys. 2018; 19(5): 506-516, doi: $10.1002 / \mathrm{acm} 2.12409$, indexed in Pubmed: 29978548.

9. Johansen S, Fosså K, Nesvold IL, et al. Arm and shoulder morbidity following surgery and radiotherapy for breast cancer. Acta Oncol. 2014; 53(4): 521-529, doi: 10.3109/0 284186X.2014.880512, indexed in Pubmed: 24495044.

10. Kapanen M, Laaksomaa M, SkyttäT, et al. Residual position errors of lymph node surrogates in breast cancer adjuvant radiotherapy: Comparison of two arm fixation devices and the effect of arm position correction. Med Dosim. 2016; 41(1): 47-52, doi: 10.1016/j.meddos.2015.08.001, indexed in Pubmed: 26482907.

11. Shah AP, Dvorak T, Curry MS, et al. Clinical evaluation of interfractional variations for whole breast radiotherapy using 3-dimensional surface imaging. Pract Radiat Oncol. 2013;3(1): 16-25, doi: 10.1016/j.prro.2012.03.002, indexed in Pubmed: 24674259.

12. Jimenez RB, Batin E, Giantsoudi D, et al. Tattoo free setup for partial breast irradiation: A feasibility study. J Appl Clin Med Phys. 2019; 20(4): 45-50, doi: 10.1002/acm2.12557, indexed in Pubmed: 30945803.

13. Seppälä J, Vuolukka K, Virén T, et al. Breast deformation during the course of radiotherapy: The need for an additional outer margin. Phys Med. 2019; 65: 1-5, doi: 10.1016/j. ejmp.2019.07.021, indexed in Pubmed: 31430580.

14. Padilla L, Kang $\mathrm{H}$, Washington $\mathrm{M}$, et al. Assessment of interfractional variation of the breast surface following conventional patient positioning for whole-breast radiotherapy. J Appl Clin Med Phys. 2014; 15(5): 4921, doi: 10.1120/ jacmp.v15i5.4921, indexed in Pubmed: 25207578.

15. VisionRT. ROI-guidelines, user manual.

16. Russell C, Mack H, Paul S, et al. OC-0190: Surface guided radiation therapy for breast cancer improves accuracy without the need for skin marks. Radiother Oncol. 2018; 127: S102, doi: 10.1016/s0167-8140(18)30500-0.

17. Hattel SH, Andersen PA, Wahlstedt IH, et al. Evaluation of setup and intrafraction motion for surface guided whole-breast cancer radiotherapy. J Appl Clin Med Phys. 2019; 20(6): 39-44, doi: 10.1002/acm2.12599, indexed in Pubmed: 31187538.

18. Cravo Sá A, Fermento A, Neves D, et al. Radiotherapy setup displacements in breast cancer patients: 3D surface imaging experience. Rep Pract Oncol Radiother. 2018; 23(1): 61-67, doi: 10.1016/j.rpor.2017.12.007, indexed in Pubmed: 29379398.

19. Alderliesten T, Sonke JJ, Betgen A, et al. Application of $3 \mathrm{D}$ surface imaging in breast cancer radiotherapy. Medical Imaging 2012. Proceedings of the SPIE. 2012; 8316, doi: $10.1117 / 12.911242$.

20. Jensen CA, Acosta Roa AM, Lund JÅ, et al. Intrafractional baseline drift during free breathing breast cancer radiation therapy. Acta Oncol. 2017; 56(6): 867-873, doi: 10.1080/0 284186X.2017.1288924, indexed in Pubmed: 28464748.

21. Skyttä T, Kapanen M, Laaksomaa M, et al. Improving the reproducibility of voluntary deep inspiration breath hold technique during adjuvant left-sided breast cancer radiotherapy. Acta Oncol. 2016; 55(8): 970-975, doi: 10.3109/0 284186X.2016.1161823, indexed in Pubmed: 27070120.

22. Laaksomaa M, Sarudis S, Rossi M, et al. AlignRT and Catalyst ${ }^{\mathrm{TM}}$ in whole-breast radiotherapy with DIBH: Is IGRT still needed? J Appl Clin Med Phys. 2019; 20(3): 97-104, doi: 10.1002/acm2.12553, indexed in Pubmed: 30861276. 